\title{
Direct detection of a magnetic field at the surface of V390 Aurigae - an effectively single active giant ${ }^{\star}$
}

\author{
R. Konstantinova-Antova ${ }^{1}$, M. Aurière ${ }^{2}$, I. Kh. Iliev ${ }^{1}$, R. Cabanac $^{2}$, J.-F. Donati ${ }^{2}$, D. Mouillet $^{3}$, and P. Petit ${ }^{2}$ \\ 1 Institute of Astronomy, Bulgarian Academy of Sciences, 72 Tsarigradsko shose, 1784 Sofia, Bulgaria \\ e-mail: [antovi;iliani]@astro.bas.bg \\ 2 Laboratoire d'Astrophysique de Toulouse-Tarbes, Université Paul Sabatier, CNRS, Observatoire Midi Pyrénées, \\ 57 avenue d'Azereix, 65008 Tarbes, France \\ e-mail: [michel.auriere;rcabanac; donati;petit]@ast.obs-mip.fr \\ ${ }^{3}$ Laboratoire d'Astrophysique, CNRS, Observatoire de Grenoble, BP 53, 38041 Grenoble Cedex 09, France \\ e-mail: david.mouillet@obs.ujf-grenoble.fr
}

Received 19 July 2007 / Accepted 8 December 2007

\section{ABSTRACT}

\begin{abstract}
Aims. We have studied the active giant V390 Aur using spectropolarimetry to obtain direct and simultaneous measurements of the magnetic field and the activity indicators in order to infer the origin of the activity.

Methods. We used the new spectropolarimeter NARVAL at the Bernard Lyot Telescope (Observatoire du Pic du Midi, France) to obtain a series of Stokes $I$ and Stokes $V$ profiles. Using the LSD technique we were able to detect the Zeeman signature of the magnetic field in each of our 5 observations and to measure its longitudinal component. Using the wide wavelength range of the spectra we could monitor the CaII K\&H and IR triplet, as well as the $\mathrm{H}_{\alpha}$ lines which are activity indicators. The Stokes I LSD profiles enabled us to detect and measure the profiles of two weak stellar companions.

Results. From five observations obtained from November 2006 to March 2007, we deduce that the magnetic field has a complex structure which evolves with time and is reminiscent of a dynamo-induced magnetic field. The activity indicators also present day to day variations, but their behaviour does not completely follow the magnetic field variations, because their longitudinal component can cancel the contribution of complex magnetic features. There is a significant difference between the magnetic field observed on November 27, 2006 and on March 15, 2007, at the same rotational phase, but with an interval of 10 rotations. The behaviour of the activity indicators together with the measured enhanced magnetic field on March 15, 2007 support the idea of a change in the field topology.

Analysis (RV and $E W$ ) of the absorption components of the Stokes $I$ LSD profile shows that the secondary of the visual wide orbit binary ADS 3812 is itself a spectroscopic binary, and suggests that the synchronization effect does not play role for V390 Aur (the primary), and that the giant should be considered as effectively single with regard to its fast rotation and activity.
\end{abstract}

Key words. stars: magnetic fields - stars: activity - stars: binaries: general

\section{Introduction}

During the $90 \mathrm{~s}$ and more recently, observations have detected magnetic activity in single late giants of small and intermediate mass at different evolutionary stages. Most of these stars rotate faster than the average of their spectral and luminosity classes (De Medeiros et al. 1996). A dynamo is generally considered as operating in evolved single stars although the exact reasons for their fast rotation and conditions for dynamo operation are not completely understood yet. One possible explanation is angular momentum dredge-up during the first ascent on the Red Giant Branch (RGB) (Simon \& Drake 1989). Another one is planet engulfment at the same evolutionary stage (Siess \& Livio 1999).

Older observations of some giants (Hubrig et al. 1994) gave $3 \sigma$ accuracy measurements of the longitudinal magnetic field that were not considered as definitive magnetic field detection. The magnetic field has been recently detected by spectropolarimetry in the case of the FK Com-type giant HD 199178 (a possible merger, Petit et al. 2004), and in RS CVn-type giants (synchronized binaries, Berdyugina et al. 2006).

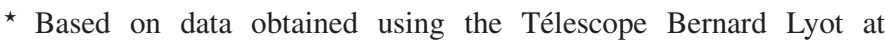
Observatoire du Pic du Midi, CNRS and Université Paul Sabatier, France.
V390 Aur $=$ HD 33798 is a G8 III star of about $2 M_{\odot}$, classified as a chromospherically active giant by Fekel \& Marshall (1991). They have reported moderately strong CaII K\&H emission cores, periodic photometric variability $(P=9.825 \mathrm{~d})$ and fast rotation $\left(v \sin i=29 \mathrm{~km} \mathrm{~s}^{-1}\right)$ for this star. A radial velocity study by the same authors concluded that V390 Aur is a single star.

Later studies of V390 Aur in the X-ray (Huensch et al. 1998; Gondoin 1999, 2003) and in the optical (Konstantinova-Antova et al. 2000; Konstantinova-Antova et al. 2005) revealed a high Xray luminosity of about $10^{30} \mathrm{erg} \mathrm{cm}^{-2} \mathrm{~s}^{-1}$, and optical flares. In the present study we obtained, for the first time, direct evidence of a magnetic field in this active giant at several phases of its rotational period, and we simultaneously followed the behaviour of the spectral line activity indicators CaII $\mathrm{K} \& \mathrm{H}, \mathrm{H}_{\alpha}$ and the CaII IR triplet.

V390 Aur is the primary of the visual binary ADS 3812 and its secondary is only 0.358 arcsec away. According to Hipparcos, the secondary is 1.85 mag fainter. As a result, the components of this visual binary were observed together in the aperture of both previous spectral observations and ours. A detailed discussion on this point is presented in Konstantinova-Antova et al. (2005) and in the present paper. 
Table 1. $R_{\mathrm{c}}$ values for CaII $\mathrm{K}, \mathrm{H}_{\alpha}$ and $B_{1}$ values for the magnetic field. Phase is regarding $9.825 \mathrm{~d}$ period considered as the rotational period of V390 Aur.

\begin{tabular}{cccccc}
\hline \hline Date & Phase & $\begin{array}{c}\text { CaII K } \\
V \text { comp. }\end{array}$ & $\begin{array}{c}\text { CaII K } \\
R \text { comp. }\end{array}$ & $\mathrm{H}_{\alpha}$ & $\begin{array}{c}B_{1} \\
\text { Gauss }\end{array}$ \\
\hline 27 Nov. 06 & 0.00 & 0.498 & 0.659 & 0.412 & -5 \\
29 Nov. 06 & 0.21 & 0.460 & 0.612 & 0.414 & -15 \\
30 Nov. 06 & 0.31 & 0.511 & 0.669 & 0.398 & -12 \\
11 Mar. 07 & 0.50 & 0.513 & 0.661 & 0.344 & -9 \\
15 Mar. 07 & 0.98 & 0.551 & 0.691 & 0.397 & -15 \\
\hline
\end{tabular}

A sinusoidal photometric variation with a 9.8 day period was determined by Spurr \& Hoff (1987) and refined to 9.825 days by Hooten \& Hall (1990). We consider this period as the rotational period of V390 Aur because it is near the 11.6 day maximum period calculated under a solid rotation assumption ( $P_{\text {rot }}<50.6 R / v \sin i$, where $P_{\text {rot }}$ is the period in days, $R$ is the computed stellar radius in sun radius units, and $v \sin i$ is the projected radial velocity in $\mathrm{km} \mathrm{s}^{-1}$ ), obtained using $v \sin i$ value reported in Fekel \& Marshall (1991) and the radius modeled by Gondoin (2003). In this case the inclination angle of the axis of rotation is about $56 \mathrm{deg}$.

\section{Observations and methods}

The observations of V390 Aur were performed at the 2-m Bernard Lyot Telescope (TBL) of Pic du Midi with NARVAL, a new generation spectropolarimeter (Aurière 2003). We observed the star 3 times in November 2006 during the science demonstration time of the instrumentation, and 2 times in March 2007 (Table 1). NARVAL is a copy of the instrument ESPaDOnS installed at the CFHT at the end of 2004 (Donati et al. 2007). NARVAL is a fiber-fed echelle spectrometer allowing the whole spectrum from $370 \mathrm{~nm}$ to $1000 \mathrm{~nm}$ to be recorded in each exposure, in 40 orders aligned in the CCD frame by 2 crossdisperser prisms. NARVAL was used in polarimetric mode with a spectral resolution of about 65000 . Stokes $I$ (unpolarised) and Stokes $V$ (circular polarization) parameters were obtained by 4 sub-exposures between which the retarders, Fresnel rhombs, were rotated in order to exchange the beams in the instrument and to reduce spurious polarization signatures. During the technical tests and science demonstration time, magnetic and non magnetic stars were observed which showed that NARVAL worked properly and was 30 times more efficient than the previous instrument MuSiCoS (Baudrand \& Boehm 1992; Donati et al. 1999). The total exposure time was $1200 \mathrm{~s}$ on Nov. 27 , 2006 and $2400 \mathrm{~s}$ for all other observations. The extraction of the spectra was performed using Libre-ESpRIT (Donati et al. 1997, 2007), a fully automatic reduction package installed at TBL. For the Zeeman analysis, least-square deconvolution analysis (LSD, Donati et al. 1997) was applied to all observations. We used a mask calculated for an effective temperature of $5000 \mathrm{~K}$, $\log g=3.0$ and a microturbulence of $2.0 \mathrm{~km} \mathrm{~s}^{-1}$, consistent with physical parameters given by Gondoin (2003). In the present case this method enabled us to average about 12700 lines and to get Stokes $I$ and Stokes $V$ profiles with greatly improved signal-to-noise ratio $(\mathrm{S} / \mathrm{N})$. A significant Zeeman Stokes $V$ signal was detected for each observation. The null spectrum given by the standard procedure (Donati et al. 1997) was also examined, which showed no signal. We then computed the longitudinal magnetic field $B_{1}$ in $\mathrm{G}$, using the first-order moment method (Donati et al. 1997; Rees \& Semel 1979). To infer $B_{1}$ values, we
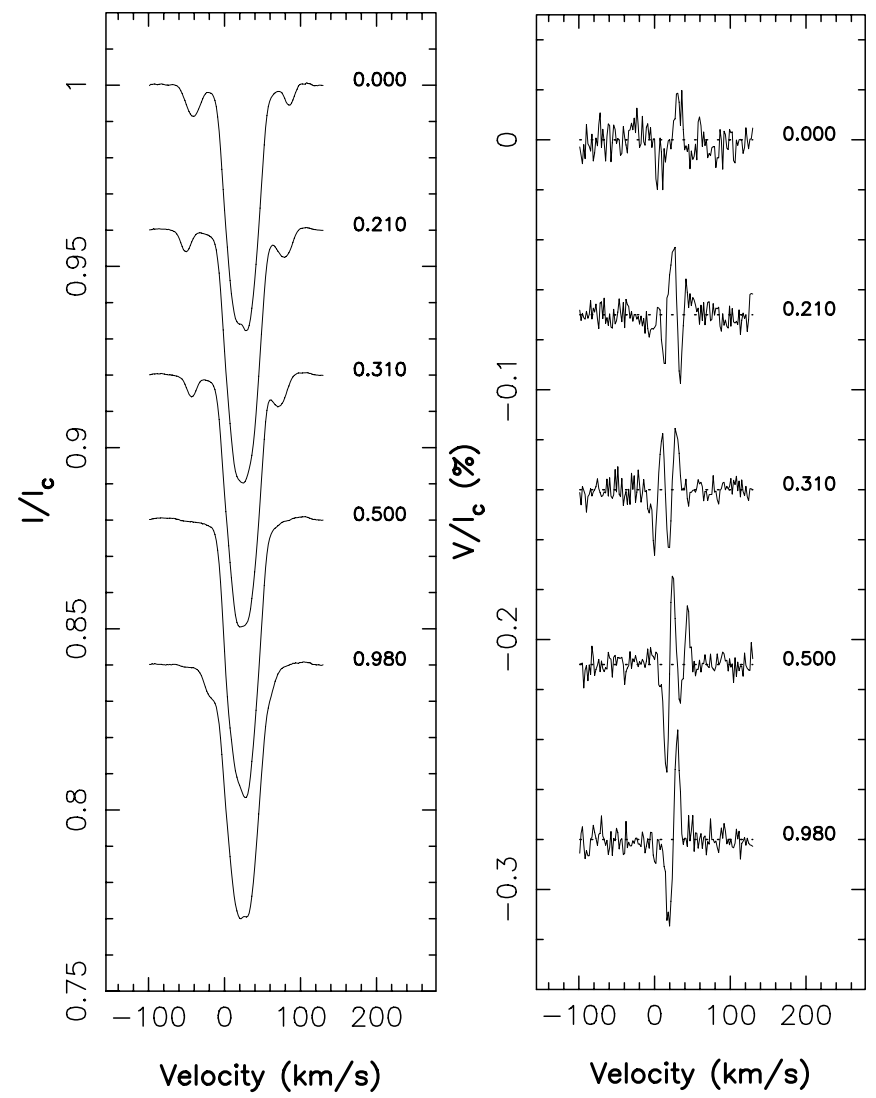

Fig. 1. LSD profiles obtained for our 5 observations of V390 Aur. Left: Stokes $I$ profiles. Right: Stokes $V$ profiles (the dotted lines illustrate the zero level of each profile). Rotational phase is indicated to the right of each profile. Successive profiles are shifted vertically for display purposes. The weak absorption features observed during rotational phases $0.00-0.310$ will be considered in detail in Sect. 3.2.

integrated the LSD profiles between -17 and $+66 \mathrm{~km} \mathrm{~s}^{-1}$, which avoid significant blending by the faint companion profiles.

The activity of the star for the same period has been monitored with measurements of the relative intensity regarding the continuum $\left(R_{\mathrm{c}}\right)$ for the line-activity indicators CaII $\mathrm{K} \& \mathrm{H}$, the CaII IR triplet and $\mathrm{H}_{\alpha}$. The $\mathrm{S} / \mathrm{N}$ is greater than 50 for the CaII K\&H emisssion cores and greater than 500 for the rest of the spectral lines mentioned above.

\section{Results and discussion}

\subsection{Magnetic field and the behaviour of the activity indicators}

Our 5 Stokes $V$ profiles presented in Fig. 1 show clear Zeeman polarization signatures. Following the quantitative criterion of Donati et al. (1997) the 5 detections are definite ones. These profiles are both complex and variable. The Stokes $I$ profiles in the November observations show 2 weak absorption profiles which correspond to faint companions and which will be analysed in Sect. 3.2. Figure 1 shows clearly that the magnetic field signature is associated with the main profile which is that of the giant V390 Aur. The associated longitudinal magnetic field measurements $B_{1}$ vary in the interval $-5--15 \mathrm{G}$ for the entire observation interval. The $B_{1}$ accuracy (one $\sigma$ ) is $\pm 5 \mathrm{G}$ for the first night, $\pm 4 \mathrm{G}$ for the last one, and $\pm 3 \mathrm{G}$ for all others. The data for $B_{1}$ are presented in Table 1. 

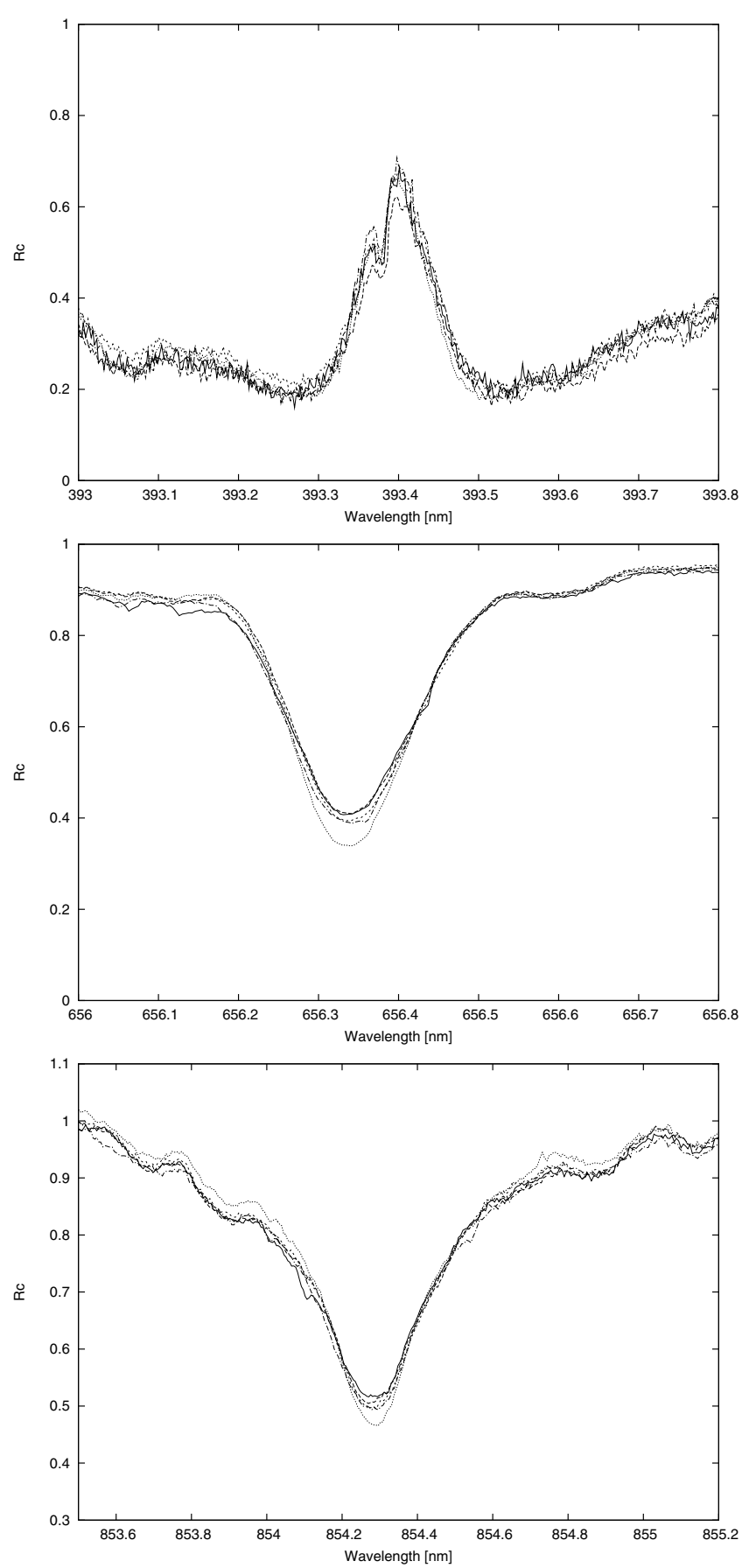

Fig. 2. CaII K, $\mathrm{H}_{\alpha}$ and CaII IR 8542 A behaviour for V390 Aur.

These observations give the first direct detection of a magnetic field in an active giant that could be considered as an effectively single one with regard its fast rotation and activity.

The parallel analysis of the CaII K\&H and IR triplet as well as the $\mathrm{H}_{\alpha}$ shows variability, too. The data for $R_{\mathrm{c}}$ of the CaII K and $\mathrm{H} \alpha$, also presented in Table 1 and Fig. 2 show the variability of these lines.

No one-to-one correlation between the magnetic field variations and the behaviour of the activity indicators was found. This may be due to the fact that $B_{1}$ measurements correspond to an integration of the longitudinal magnetic field over the stellar disk and that magnetic features with opposite signs may cancel their contributions. For the CaII K\&H emission cores we found that
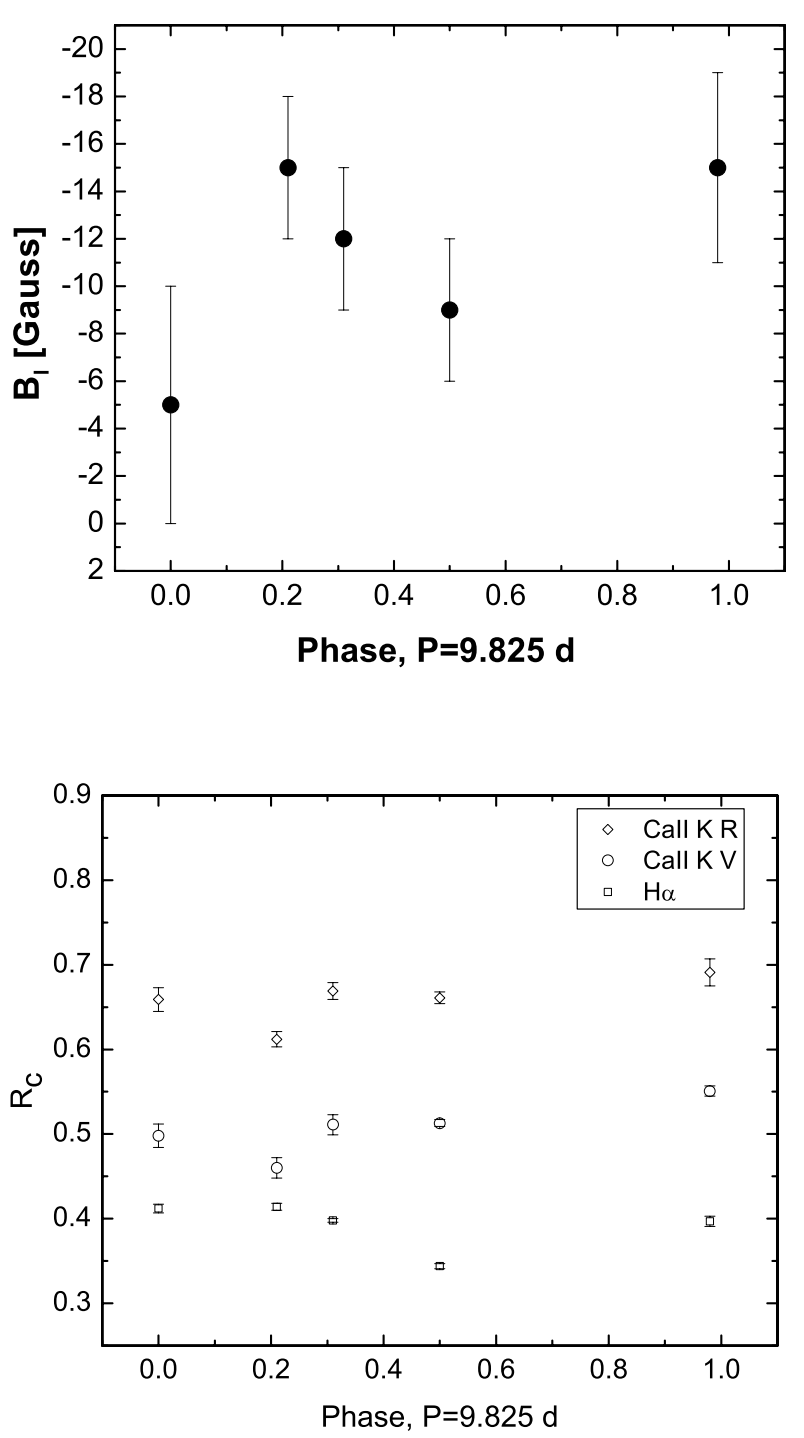

Fig. 3. Magnetic field (upper panel) and the bahaviour of the activity indicators (lower pannel) for V390 Aur for rotational period $P=9.825 \mathrm{~d}$. An inverse $Y$-axis is used for $B_{1}$ to illustrate the strength of the field.

the emission is resolved into blue $(V)$ and red $(R)$ components in both $\mathrm{K}$ and $\mathrm{H}$ lines and both exhibit identical variability behaviour. For the H\&K lines in all our spectra, we measured the relative intensity ratio $V / R<1$. Such an asymmetry indicates upward motions and possible mass outflow in the low chromosphere, where these lines are formed. The $V / R$ ratio also exhibits slight variability.

The phase-dependant behaviour of the magnetic field and of the activity indicators are presented in Fig. 3. We have not covered the whole rotational period yet, and between the 3 first measurements (November 2006 observations) and the two last ones (March 2007 observations) there are 10 rotations. We have obtained two observations very close in phase (November 27 , 2006 and March 15, 2007) for this period. There is a significant change in the Stokes $V$ profile and a variation of the $B_{1}$ measurement between both observations. On the first date we detected a weak longitudinal magnetic field $(-5 \mathrm{G})$, while on March 15 , 2007 we detected a value of $-15 \mathrm{G}$. On the second date, the CaII $\mathrm{K}$ intensities are the highest ones for the whole dataset. These variations together with the complex Stokes $V$ profile 
Table 2. Astrometric measurements of ADS $3812=$ A 1031, described in Sect. 3.2.1.

\begin{tabular}{cccc}
\hline \hline Epoch & $\theta(\mathrm{deg})$ & $\rho(\operatorname{arcsec})$ & Method \\
\hline 1965.991 & 43 & 0.33 & micrometer \\
1970.129 & 37 & 0.40 & micrometer \\
1991.25 & 80 & 0.36 & Hipparcos \\
1999.831 & 143.1 & 0.736 & speckle interf. \\
\hline
\end{tabular}

observed, on November 29, 2006 for example, show that a dynamo driven magnetic field is at work, rather than a large-scale geometrically stable magnetic field, which is supposed to exist at the surface of the slowly rotating active giant EK Eri (Stepien 1993; Strassmeier et al. 1999).

In general, the $\mathrm{H}_{\alpha}$ behaviour is complex, due to the fact that it consists of a photospheric absorption partially filled-in by a chromospheric emission component. For V390 Aur, the emission component is weak, and the photospheric absorption dominates. This fact is due to the lower density and gravity in the atmosphere of RGB stars, compared with those in active dwarfs (Cram \& Mullan 1985). Similar $\mathrm{H}_{\alpha}$ behaviour is also reported for other active single giants (Fekel \& Balachandran 1993).

While the CaII $\mathrm{H}$ and $\mathrm{K}$ variations appear erratic in some ways, the variations of $\mathrm{H}_{\alpha}$ are smoother and linked to rotational modulation due to spots. Even though the emission component is weak, it also plays a role in the variability of $\mathrm{H}_{\alpha}$. For example, on March 15, 2007, when a stronger magnetic field was detected, one would also expect a deeper photospheric absorption. However, the emission component should also be stronger then, and has filled-in the absorption profile more resulting in the relative intensity of $\mathrm{H}_{\alpha}$ (Fig. 3). This behavior is in agreement with the observed CaII emission and also supports the conclusion that after 10 rotations the topology of the active areas in this hemisphere of the giant has been changed. The CaII IR triplet lines have similar structure and behaviour as $\mathrm{H}_{\alpha}$.

In the future, we need better phase coverage to be able to refine our knowledge about the magnetic field and the variation of the activity indicators with rotational period in this giant.

\subsection{V390 Aur as the primary component of a multiple system}

\subsubsection{ADS 3812}

V390 Aur is the primary of the visual binary ADS 3812. Four measurements of this binary have been published and concern 4 epochs: Couteau (1967), Van Biesbroeck (1974), Perryman et al. (1997), Mason et al. (2001). Table 2 gives the measurements. Three different techniques were used, they show a rotation of the secondary of about 20 degrees every 10 years in the interval 1965-2000. This means that ADS 3812 is a physical binary and that the expected orbital period is greater than a century.

Recently, the distance was determined by Hipparcos when the separation between the two components was 0.36 arcsec: $111.85 \mathrm{pc}$ distance gives about 40 a.u. separation. The mass of V390 Aur is estimated to be $1.8 M_{\odot}$ (Gondoin 2003). The secondary has been estimated as G1V (Stephenson\& Sandwall 1969), or an F dwarf or a subgiant (Konstantinova-Antova et al. 2005) and these authors suggest a mass of $1.3 M_{\odot}$. With this last assumption and a circular orbit hypothesis, KonstantinovaAntova et al. (2005) estimated a 140 year orbital period for ADS 3812. This implies, again assuming a circular orbit, that the differential radial velocity between the 2 components of
Table 3. Radial velocity measurements $\mathrm{RV}\left(\mathrm{km} \mathrm{s}^{-1}\right)$ and Equivalent

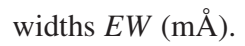

\begin{tabular}{ccccccc}
\hline \hline Date & $R V_{\mathrm{A}}$ & $R V_{\mathrm{b}}$ & $R V_{\mathrm{c}}$ & $E W_{\mathrm{A}}$ & $E W_{\mathrm{b}}$ & $E W_{\mathrm{c}}$ \\
\hline 27 Nov. 06 & 23.2 & -40.8 & 83.8 & 108 & 11 & 7 \\
29 Nov. 06 & 23.5 & 75.5 & -50.5 & 107 & 13 & 6 \\
30 Nov. 06 & 23.6 & 69.7 & -43.05 & 107 & 13 & 6 \\
11 Mar. 07 & 23.4 & & & & & \\
15 Mar. 07 & 23.5 & & & & & \\
\hline
\end{tabular}

ADS 3812 is smaller than $1 \mathrm{~km} \mathrm{~s}^{-1}$. Both stars are in the aperture of NARVAL, and because $v \sin i$ of V390 Aur is $29 \mathrm{~km} \mathrm{~s}^{-1}$ (Fekel \& Marschall 1991) both spectra are blended. Thus, if the secondary is a single star, one would expect that the observed spectrum is mainly that of V390 Aur which is brighter than its companion by 2-3 mag (1.85 mag from Hipparcos; 3 mag from visual observers).

\subsubsection{Two faint companions?}

V390 Aur was observed 5 times with NARVAL. During three November observations, two features of faint objects were observed in the Stokes I LSD profiles. These features are invisible on March 11 and one appears in the blue wing of the main profile on March 15. By fitting a Gaussian function to the individual features we measured their radial velocities and equivalent widths. Table 3 gives the measurements of $R V$ and $E W$ for the 3 objects $\mathrm{A}, \mathrm{b}$, and $\mathrm{c}$ (the main profile, the blue wing profile, and the red wing profile) on November 27. Visual inspection of Fig. 1 and $E W$ measurements suggest that $\mathrm{b}$ and c exchanged places on November 29 and 30 with respect to November 27.

The errors of the individual components, derived by varying of the fitting conditions of the Gaussian function, are estimated to be $0.1 \mathrm{~km} \mathrm{~s}^{-1}$ for $\mathrm{A}$ and $\mathrm{b}, 0.5 \mathrm{~km} \mathrm{~s}^{-1}$ for $\mathrm{c}$. The standard error for $E W$ is about $1.5 \mathrm{~m} \AA$.

Interpreting the data in Table 3 depends on whether we make the hypothesis that $\mathrm{b}$ and $\mathrm{c}$ are close companions orbiting the primary or correspond to the secondary star.

\subsubsection{V390 Aur as a tight triple star}

Fekel \& Marschall (1991) performed an accurate study of radial velocity variations of V390 Aur, using about 40 measurements obtained from 6 instruments and spanning about 3 years. They did not find any periodic variations using either all the data together or some homogeneous subsamples. They concluded that the giant V390 Aur is a single star with a radial velocity of $22.5 \pm 0.2 \mathrm{~km} \mathrm{~s}^{-1}$. From our 5 measurements we obtain for A an average radial velocity of $23.44 \pm 0.08 \mathrm{~km} \mathrm{~s}^{-1}$. The difference between the two average values can be due to different calibrations and our measurements are thus consistent with the results of Fekel \& Marschall (1991).

Let us assume that objects $\mathrm{b}$ and $\mathrm{c}$ are gravitationally bound to A. Table 3 shows that the radial velocities of $\mathrm{b}$ and $\mathrm{c}$ varied by more than $5 \mathrm{~km} \mathrm{~s}^{-1}$ per day, whereas the radial velocity of A is measured to increase in only a few tens of $\mathrm{km} \mathrm{s}^{-1}$ per day. To obtain the mass ratios, we alternatively consider $\mathrm{A}, \mathrm{b}$ and $\mathrm{A}, \mathrm{c}$ as isolated binaries, since A would evidently dominate the system. In the case of spectroscopic binaries in which both spectra are visible, Wilson (1941) showed that the mass ratio of the components is always equal to the ratio of the (inverse) ratio of radial velocities to the radial velocity of the system. Using the maximum difference in radial velocities observed and reported in 
Table 3 and Wilson's formula, we find that the objects $\mathrm{b}$ and $\mathrm{c}$ are about 30 times lighter than A. Taking the total mass of V390 Aur as $1.8 M_{\odot}$ (Gondoin 2003) gives mass estimates for $\mathrm{b}$ and $\mathrm{c}$ as less than $0.06 M_{\odot}$. Thus, these objects would be brown dwarfs and $10^{-4}$ to $10^{-5}$ fainter than the sun (Baraffe et al. 1998). Such objects should be invisible in our spectra, in contradiction to the properties of $\mathrm{b}$ and $\mathrm{c}$ components we observed.

\subsubsection{The secondary of ADS 3812 as a spectroscopic binary}

The alternative scenario is to consider that $\mathrm{b}$ and $\mathrm{c}$ belong to the secondary of ADS 3812. Using the relation of Wilson (1941) and the data from Table 3, a mass ratio $M b / M c=1.15$ has been determined. The rough estimate of the magnitude difference between $A$ and $b+c$, based our equivalent width measurements in Table 3 is 1.9 mag and is consistent with the difference between the components of ADS 3812 measured by Hipparcos (this supposes that all 3 stars have the same temperature and spectrum. We used an LSD Stokes $I$ profile with a mask calculated for an effective temperature of $7000 \mathrm{~K}$ and $\log g=4$ to mimic an FV companion, see Sect. 3.2.1. We then measured the $E W$ of the 3 profiles and deduced that the difference in spectral type between V390 Aur and its companion does not hamper our conclusion).

\subsubsection{ADS 3812 again}

The simplest interpretation of our series of LSD Stokes $I$ profiles is that ADS 3812 is a multiple system: the primary is the magnetically active giant V390 Aur, and the secondary is a double line spectroscopic binary. To refine the properties of the secondary system more measurements are necessary.

For the primary, V390 Aur, our results together with the large orbital period of the secondary support the idea that the synchronization effect does not play a role in its fast rotation, and the star should be considered as an effectively single giant with respect to its magnetic activity.

\section{Conclusions}

1. We have directly detected the presence and variability of a magnetic field in the RGB star V390 Aur. The magnetic field varies along the rotational phase of $9.825 \mathrm{~d}$. On March 15, 2007 a value of $-15 \mathrm{G}$ was measured for the longitudinal magnetic field whereas it was only of $-5 \mathrm{G}$ at the same phase, on November 27, 2006, 10 rotations before. We interpret this fact as a change in the field topology.

2. The variations of the activity indicators CaII $\mathrm{K} \& \mathrm{H}, \mathrm{CaII}$ IR triplet and $\mathrm{H}_{\alpha}$ do not closely follow the longitudinal magnetic field variations since it is an averaged value on the stellar disk and magnetic features of opposite polarity may cancel each other. On March 15, 2007 their behaviour could be explained in terms of a stronger emission component from the more active chromosphere and supports the idea that after
10 rotations the magnetic field topology in this hemisphere has been changed.

3. The analysis of our series of LSD profiles revealed that ADS 3812 is a multiple system. The most likely explanation is that the primary is the active giant V390 Aur, and the secondary is a double line spectroscopic binary, in a wide orbit around the primary. Our observations confirm that synchronization does not play a role in the system and the giant could be considered as effectively single with regard to the reasons for its fast rotation and magnetic activity.

The complex behaviour of V390 Aur demands a complete suite of observations spanning a full rotation period. These observations would allow a better monitoring of the magnetic field variations in parallel with the traditional activity indicators, and also would refine the properties of the multiple system ADS 3812.

Acknowledgements. I.I. acknowledges the partial support from Bulgarian NSF under grant F-1403/2004. We warmly thank the NARVAL project team and the TBL team for making it possible to obtain the data used in this paper during the scientific demonstration time. We acknowledge the use of the Hipparcos database.

\section{References}

Aurière, M. 2003, in Magnetism and Activity of the Sun and Stars, ed. J. Arnaud, \& N. Meunier, EAS Publ. Ser., 9, 105

Baraffe, I., Chabrier, G., Allard, F., \& Hauschildt, P. H. 1998, A\&A, 337, 403

Baudrand, J., \& Boehm T. 1992, A\&A, 259, 711

Berdyugina, S. V., Petit, P., Fluri, D. M., et al. 2006, ASPC, 358, 381B

Couteau, P. 1967, JO, 50, 41

Cram, L. E., \& Mullan, D. J. 1985, ApJ, 294, 626

De Medeiros, J. R., Da Rocha, C., \& Mayor, M. 1996, A\&A, 314, 499

Donati, J.-F., Semel, M., Carter, B. D., et al. 1997, MNRAS, 291, 658

Donati, J.-F., Catala, C., Wade, G. W., et al. 1999, A\&A, 134, 149

Donati, J. -F., et al. 2007, in preparation

Fekel, F. C., \& Marschall, L. A. 1991, AJ, 102, 1439

Fekel, F. C., \& Balachandran, S. 1993, ApJ, 403, 708

Gondoin, P. 1999, A\&A, 352, 217

Gondoin, P. 2003, A\&A, 404, 355

Hooten, J. T., \& Hall, D. S. 1990, ApJS, 74, 225

Hubrig, S., Plachinda, S. I., Huensch, M., \& Schroeder, K.-P. 1994, A\&A, 291, 890

Hunsch, M., Schmitt, J. H. M. M., \& Voges, W. 1998, A\&A, 127, 251

Konstantinova-Antova, R. K., Antov, A. P., \& Bachev, R. S. 2000, IBVS, 4867

Konstantinova-Antova, R. K., Antov, A. P., Zhilyaev, B. E. et al. 2005, AN, 326, 38

Mason, B. D., Hartkopf, W. I., Holdenried, E. R., \& Rafferty, T. J. 2001, AJ, 121, 3224

Perryman, M. A. C., Lindegren, L., Kovalesky, J., et al. 1997, A\&A, 323, L49

Petit, P., Donati, J.-F., Oliveira, J. M., et al. 2004, MNRAS, 351, 826

Rees, D. E., \& Semel, M. 1979, A\&A, 74, 1

Siess, L., \& Livio, M. 1999, MNRAS, 308, 1133

Simon, T., \& Drake, S. A. 1989, ApJ, 346, 303

Spurr, A. J., \& Hoff, D. 1987, IBVS, 3028

Stephenson, C. B., \& Sandwall, N. B. 1969, AJ, 74, 689

Stepien, K. 1993, ApJ, 416, 368

Strassmeier, K. G., Stepien, K., Henry, G. W., \& Hall, D. S. 1999, A\&A, 343, 175

Van Biesbroeck, G. 1974, ApJS, 28, 413

Wilson, O. C. 1941, ApJ, 93, 29 